\title{
Surface Restructuring of Hybrid Perovskite Crystals
}

\author{
Banavoth Murali, ${ }^{\ddagger}$ Sukumar Dey, ${ }^{\ddagger}$ Ahmed L. Abdelhady, ${ }^{\dagger}$ Wei Peng, ${ }^{\ddagger}$ Erkki Alarousu, \\ Ahmad R. Kirmani, ${ }^{\ddagger}$ Namchul Cho, ${ }^{\ddagger}$ Smritakshi Phukan Sarmah, ${ }^{\ddagger}$ Manas R. Parida, ${ }^{\ddagger}$ \\ Makhsud I. Saidaminov, ${ }^{\ddagger}$ Ayan A. Zhumekenov, ${ }^{\ddagger}$ Jingya Sun, ${ }^{\ddagger}$ Mohd S. Alias, ${ }^{\text {II }}$ Emre Yengel, \\ Boon S. Ooi, ${ }^{\mathbb{T}}$ Aram Amassian, ${ }^{*}$ Osman M. Bakr, ${ }^{*}{ }^{\ddagger}$ and Omar F. Mohammed ${ }^{*}$,
}

${ }^{\ddagger}$ King Abdullah University of Science and Technology, KAUST Solar Center, Division of Physical Sciences and Engineering, Thuwal 23955-6900, Kingdom of Saudi Arabia

${ }^{\text {II }}$ King Abdullah University of Science and Technology, Photonics Laboratory, Computer, Electrical and Mathematical Sciences and Engineering Division, Thuwal 23955-6900, Kingdom of Saudi Arabia

\section{Supporting Information}

\begin{abstract}
Hybrid perovskite crystals have emerged as an important class of semiconductors because of their remarkable performance in optoelectronics devices. The interface structure and chemistry of these crystals are key determinants of the device's performance. Unfortunately, little is known about the intrinsic properties of the surfaces of perovskite materials because extrinsic effects, such as complex microstructures, processing conditions, and hydration under ambient conditions, are thought to cause resistive losses and high leakage current in solar cells. We reveal the intrinsic structural and optoelectronic properties of both pristinely cleaved and aged surfaces of single crystals. We identify surface restructuring on the aged surfaces (visualized on the atomic-scale by scanning tunneling microscopy) that lead to compositional and optical bandgap changes as well as degradation of carrier dynamics, photocurrent, and solar cell device performance. The insights reported herein clarify the key variables involved in the performance of perovskite-based solar cells and

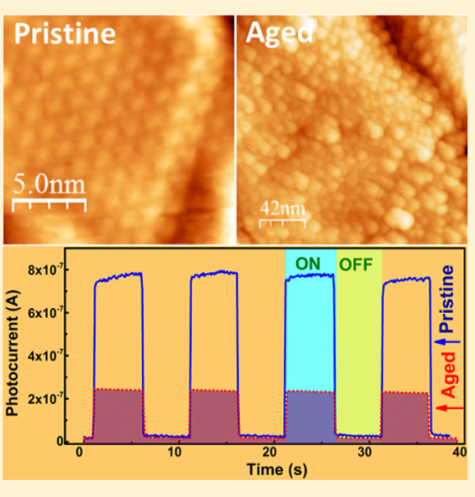
fabrication of high-quality surface single crystals, thus paving the way toward their future exploitation in highly efficient solar cells.
\end{abstract}

$\mathrm{T}$ he phenomenal performance of organic-inorganic hybrid halide perovskite materials marks the dawn of a new era in solution-processed optoelectronics and photovoltaics. ${ }^{1-4}$ Their remarkable power conversion efficiencies (PCEs) $)^{5}$ stem from unprecedented attributes, ${ }^{6}$ including their adjustable optical band gaps $^{7-9}$ high absorption coefficients, ${ }^{10}$ long electron-hole diffusion lengths, ${ }^{11}$ high carrier mobilities, ${ }^{2}$ low surface recombination velocities, ${ }^{13}$ ambipolar charge transport characteristics, and economically competitive fabrication processes, that make them particularly suited to be solution-manufactured semiconductors. ${ }^{14-17}$ These attributes have galvanized the use of organic-inorganic hybrid halide perovskite materials in applications from solar cells ${ }^{18}$ to bright light-emitting diodes, ${ }^{19}$ electrically and optically pumped lasing, ${ }^{20}$ color imaging, ${ }^{21}$ photodetectors, ${ }^{22}$ and phototransistors. $^{23}$ Their remarkable photovoltaic properties ${ }^{24,25}$ can be realized from highly ordered as well as low-density trap states. ${ }^{26}$ Optoelectronic devices are usually constructed from polycrystalline perovskite thin films, which conventionally contain a sizable density of charge traps, suggesting that device performance can be further improved by replacing polycrystalline films with single crystals that exhibit exceptionally low trap- state densities, high carrier mobility, and long carrier diffusion lengths. ${ }^{27}$ The strikingly low trap densities, commensurate with those of microelectronic-grade single-crystal silicon, suggest the substantive capability of $\mathrm{MAPbX}_{3}$ (where $\mathrm{MA}=\mathrm{CH}_{3} \mathrm{NH}_{3}{ }^{+}, \mathrm{X}=$ $\left.\mathrm{I}^{-} / \mathrm{Br}^{-} / \mathrm{Cl}^{-}\right)$perovskite single crystals, an exceptionally alluring semiconductor, compared to that of its polycrystalline film counterparts. $^{28,29}$ Given this remarkable capability, the efficient manipulation of the surface properties of single crystals is crucial to realizing high PCE.

The recent demonstration of macroscopic perovskite single crystals marks an important milestone for this material, opening new avenues for scientific inquiry as well as for engineering single crystal-based high-performance devices. However, crystal surfaces are prone to hydration under ambient conditions, ${ }^{30}$ and there are currently no methods for surface cleaning or purification that protect the crystal, thus limiting the accurate experimental estimation of its intrinsic optical and electronic properties. $^{30}$ Relative humidity $(\mathrm{RH})$ at high levels $(\geq 50 \%)$

Received: October 9, 2016

Accepted: November 6, 2016

Published: November 7, 2016 

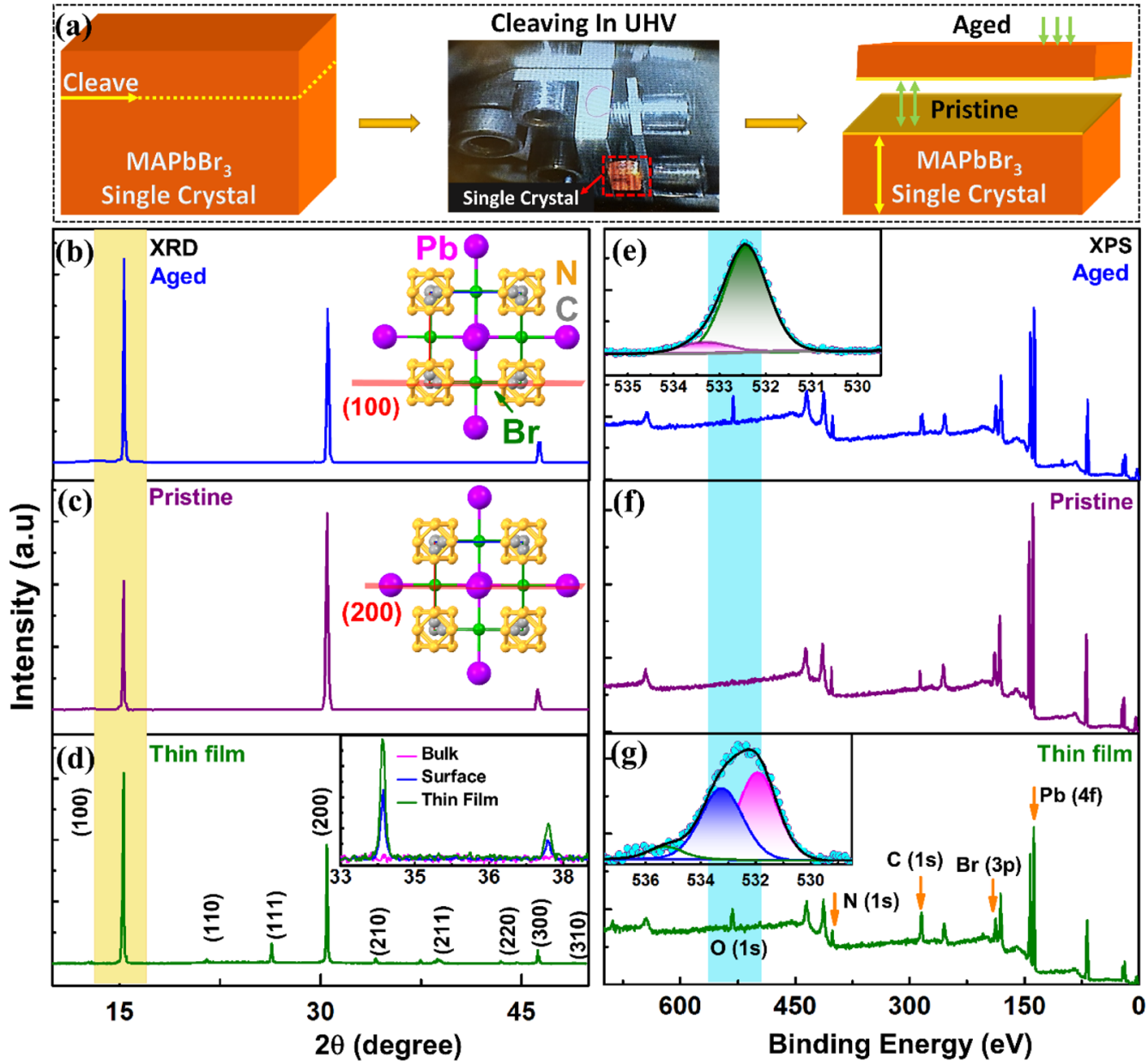

Figure 1. (a) Schematic illustration of the aged and the pristine surfaces of a single crystal upon cleaving under ultrahigh vacuum (UHV). XRD patterns and XPS spectra of $\mathrm{MAPbBr}_{3}$ perovskite: (b, e) aged surface, $(c, f)$ pristine surface, $(\mathrm{d}, \mathrm{g})$ thin film, respectively. The inset of panel $d$ shows a magnified view of the XRD patterns accounting for the weaker reflections to compare the aged surface and the thin film in the $2 \theta$ range of $33^{\circ}-39^{\circ}$. The unit cells are shown in the insets of panels $b$ and $c$ indicating the various Bragg orientations as marked. The insets of panels $e$ and $g$ show the deconvolution of the $O 1$ s peak of the aged surface and the thin film, respectively.

also has detrimental impact on the crystallization process and device performance. ${ }^{31-33}$ Of course, a single-crystal device fabricated with hydrated facets forms a poor contact with severe resistive losses and high leakage currents despite its excellent bulk properties. ${ }^{34}$ Notably, single crystals are also sensitive to various environments of moist and dry gases. ${ }^{35,36}$ Characterizing the intrinsic optoelectronic properties of a single crystal's bulk and overcoming the integration challenges associated with hydrated or degraded surfaces require a profound understanding of the characteristics of the crystal's surface and its eventual transformation under ambient conditions. ${ }^{37}$

This report focuses on mapping surface transformation of hybrid perovskite crystals and on understanding the mechanism leading to surface-structural conversion in $\mathrm{MAPbBr}_{3}$ single crystals. Our detailed optical characterizations and highresolution surface mapping show that the surface of a hybrid perovskite single crystal converts into a polycrystalline, which can extend to depths of hundreds of nanometers if left for long periods under ambient conditions. Our steady-state and timeresolved laser spectroscopy results, as well as atomic resolution microscopy data, provide decisive experimental evidence for the different structure, morphology, carrier dynamics, photocurrent, and optical band gap of the crystal's surface compared with the crystal's bulk. More specifically, scanning tunneling microscopy (STM) investigations of a hydrated crystal's surface reveal mounding due to hydration and the associated moisture uptake, whereas in situ observations of the bulk upon cleaving the crystal (Figure S1) under ultrahigh vacuum (UHV) reveal an impeccable crystal lattice, providing the real-space, atomicscale visualization of the cubic phase of an $\mathrm{MAPbBr}_{3}$ single crystal lattice at room temperature (RT). Upon exposure to ambient air, the cleaved bulk surface is shown to hydrate and reconstruct. These advances in understanding provide the fundamental basis for synthesizing high-quality perovskite single-crystal specimens for integrated photovoltaic applications.

In this Letter, we call the surface of as-grown single crystals an "aged" crystal surface, whereas we name the cleaved surface under UHV a "pristine" crystal surface. Both are schematically shown in Figure 1a. The cubic crystal structure of $\mathrm{MAPbBr}_{3}$ with a $P m \overline{3} m$ space group is identified using X-ray diffraction (XRD), as shown in Figure $1 \mathrm{~b}-\mathrm{d}$. The XRD results reveal that the Bragg reflections of aged (Figure $1 \mathrm{~b}$ ) and pristine (Figure 1c) samples show high intensity along the (100) and (200) reflections, respectively, as shown in the insets of Figures $1 \mathrm{~b}$ and $1 \mathrm{c}$. Interestingly, previously reported XRD data on single crystals with hydrated surfaces identified only the (100) orientation. $^{28,38}$ To understand the origin of the different 

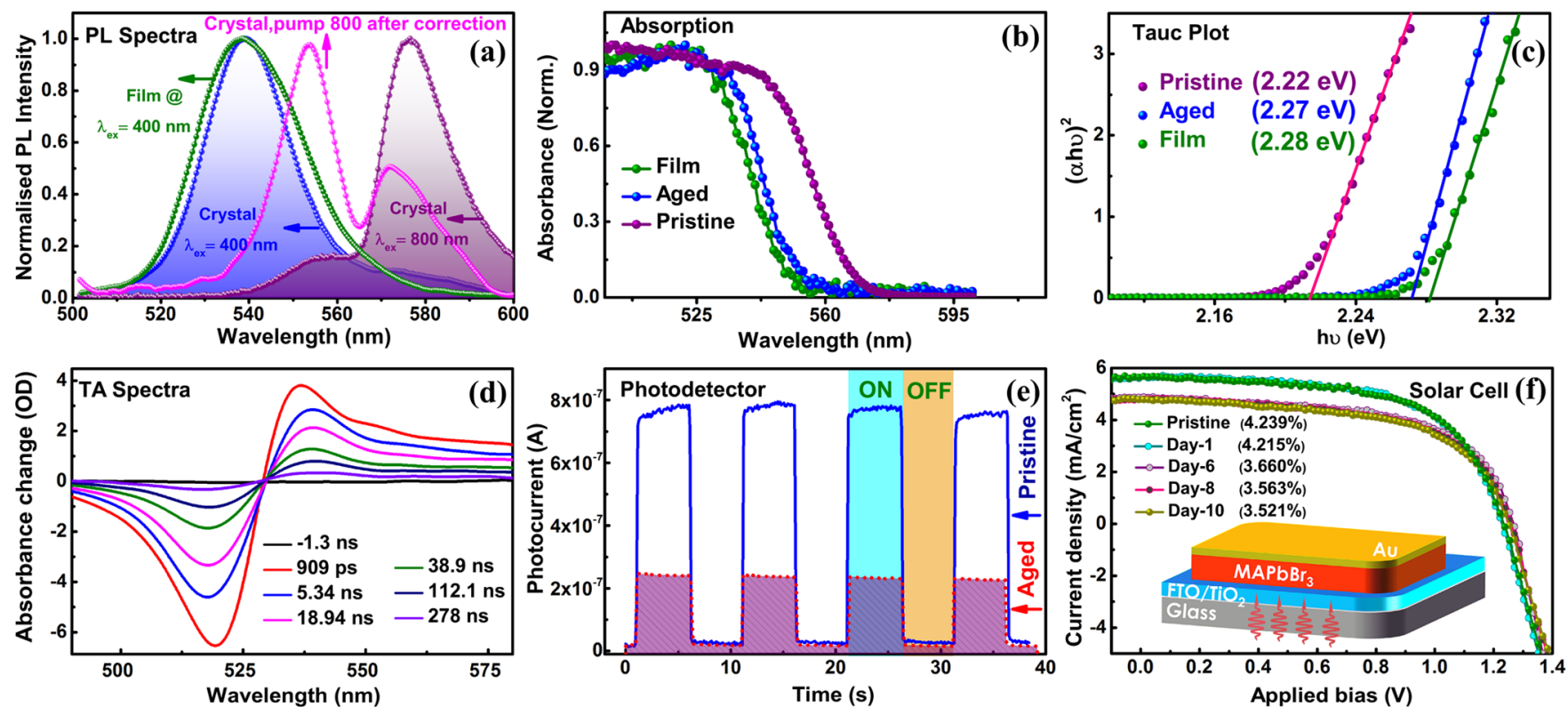

Figure 2. Comparative optical properties and device performances of $\mathrm{MAPbBr}_{3}$ single crystal: (a) PL spectra obtained at $\lambda_{\mathrm{ex}}=400 \mathrm{~nm}$, 800 $\mathrm{nm}$ and after the correction with light reabsorption; (b) absorption spectra; (c)Tauc plot showing the band gaps; (d) transient absorption spectra; (e) photoresponsive cycles under AM $1.5 \mathrm{G}$ illumination from the aged surface and pristine surface as highlighted; (f) $J-V$ curves for the device under AM 1.5 G illumination, showing the effect of hydration. The best case efficiency values are chosen to capture the effect of hydration on the device performance without prelight soaking.

intensity of the (100) and (200) planes, we conducted XRD experiments on a polycrystalline film of $\mathrm{MAPbBr}_{3}$. Surprisingly, we found that its XRD patterns resembled those of an aged surface. Our first thought was that the aged surface behaves like a polycrystalline film (Figure 1d). To further substantiate this assumption, we carried out slow-scan XRD on various crystals in the $2 \theta$ range of $33^{\circ}-39^{\circ}$ (inset of Figure 1d) in the polycrystalline film as well as on a pristine crystal and on exposed, hydrated surfaces of the aged crystal. We observed that the reflections from the (210) and (211) planes were completely absent from the pristine crystal's surface, whereas they were present on the aged crystal's surface as well as in the polycrystalline film. The difference in the intensity of the (100) and (200) reflections may be attributed to gradual homogeneous nucleation that initially drives crystal growth and to expeditious surface transformation when exposed to ambient air. This observation is in a good agreement with STM topographic images (see following discusion). Our preliminary results only indicate that (100) and (200) reflections exhibit different intensities, but it should be noted that grazingincidence wide-angle X-ray scattering (GIWAXS) can give the in-plane crystal information, ${ }^{39,40}$ which is beyond the scope of this work.

The XRD measurements confirm a characteristic of two orientations when the surface is exposed to ambient conditions, suggesting both the polycrystalline nature and the freshly cleaved pristine nature of the single crystal. We further corroborated these observations with results from several surface-sensitive experiments, including small-scale STM, photocurrent experiments, X-ray photoelectron spectroscopy (XPS), cross-sectional scanning electron microscopy (SEM), and steady-state and time-resolved electronic spectroscopy (discussed below).

We directly probed the surface composition of a single crystal and the polycrystalline film by XPS with a depth sensitivity of $\sim 5-10 \mathrm{~nm}$. The exposed aged surface of the crystal has a high content of oxygen (Figure 1e), which is completely absent from the pristine surface (Figure 1f). A principal component of $\mathrm{O} 1 \mathrm{~s}(532.0-532.5 \mathrm{eV})$ is observed on the aged surface or film and is likely to represent the $\mathrm{O}-\mathrm{H}$ and $\mathrm{O}-\mathrm{C}$ functionalities. ${ }^{41,42}$ However, the shoulder at $533.3 \mathrm{eV}$ suggests adsorbed water, ${ }^{43}$ which we confirmed conclusively using STM imaging (discussed below). The XPS measurements thus confirm that a single crystal forms a hydrated surface due to water adsorption.

Chemical composition comparisons (Table S1) of the $\mathrm{Br}: \mathrm{Pb}$ and $\mathrm{N}: \mathrm{Pb}$ ratios remained unaltered subsequent to cleaving. However, the $\mathrm{C}$ content was significantly reduced in the pristine case because of the absence of adventitious carbon contamination. XPS measurements on polycrystalline films also revealed the presence of oxygen (Figure $1 \mathrm{~g}$ and inset), providing clear experimental evidence of hydration of the surface upon exposure to ambient air.

Results from steady-state and time-resolved photoluminescence (PL) upon $1 p$ and $2 p$ excitation, absorption spectra, optical bandgap, and transient absorption of the $\mathrm{MAPbBr}_{3}$ crystal and the thin film directly distinguish the optical properties of the aged surface from those of pristine surfaces. ${ }^{33}$ We therefore exploited the penetration depths of the $1 \mathrm{P}^{44}$ (less than $200 \mathrm{~nm})$ and $2 \mathrm{P}^{45}(\geq \mu \mathrm{m})$ excitations to probe the PL of aged and bulk surfaces of the crystal. ${ }^{33}$ As can be seen in Figure $2 \mathrm{a}$, the PL spectra are centered at 540 and $580 \mathrm{~nm}$ for the $1 \mathrm{P}$ and $2 \mathrm{P}$ excitations, respectively. However, after the correction for light reabsorption using the absorption coefficient ${ }^{46}$ and diffusion length, ${ }^{38}$ the intensity of $580 \mathrm{~nm}$ peak reduces significantly and the peak at $555 \mathrm{~nm}$ become more prominent. Therefore, the peak at $580 \mathrm{~nm}$ may be a result of light reabsorption due to the very large penetration depth especially upon two-photon excitation. Further studies are in progress to fully understand the origin of these two PL peaks. We should highlight again that the PL obtained with excitation above the band gap characterizes mainly the surface layer with a thickness 

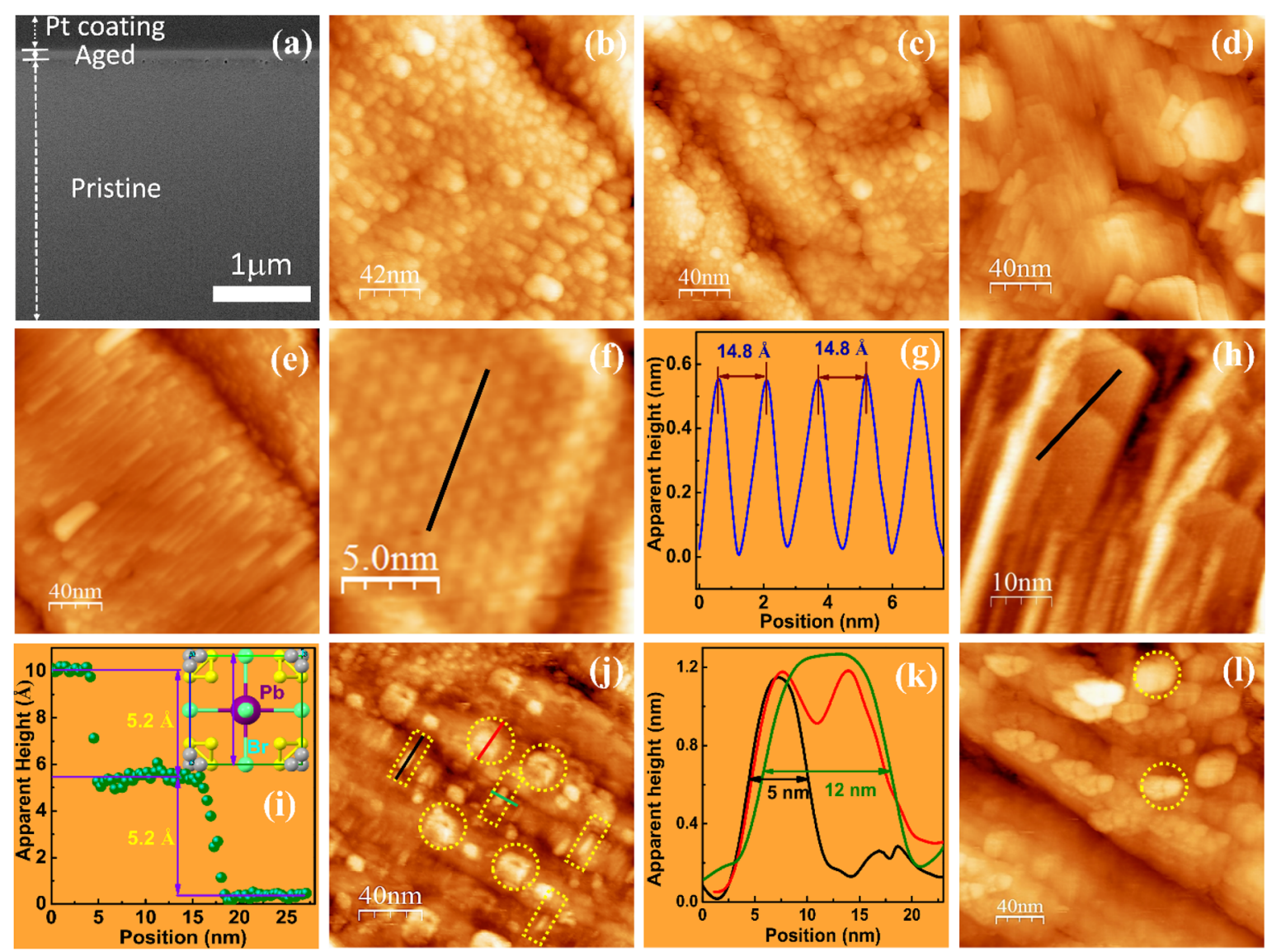

Figure 3. Real-space imaging and visualization of $\mathrm{MAPbBr}_{3}$ by SEM and STM. (a) cross-sectional FIB-SEM image showing clear contrast differences on the aged and pristine surfaces. STM images obtained from the (b, c) aged surfaces of an as-grown single crystal and (d, e) from the pristine surfaces at various positions captured at scanning parameters of $V_{b}=2.5 \mathrm{~V}$ and $I_{t}=0.7 \mathrm{nA}$. (f, h) Pristine surface showing the ordered structures with well-aligned stacked planes. $(\mathrm{g}) \mathrm{Pb}-\mathrm{Pb}$ distance along the (110) plane obtained from the height profile of panel $\mathrm{f}$. (i) Interlayer spacing measured along the dark line in panel $h$ corresponding to the lattice parameter of a cubic unit cell. ( $j, 1)$ STM images showing the hydrate formation on the pristine surface after overnight exposure to ambient air with ringlike protrusions (marked by circles) and the disunified 1D chains (marked by rectangles), indicating molecular water incorporation in the pristine surface of the single crystal. (k) Line profile on the bright line of perovskite hydrate along the length (green), diameter (black), and line profile (red) on the ring-like protrusions measured along the marked lines in panel $\mathbf{j}$.

determined by the light penetration depth, and it is normally a few hundreds of nanometers. In contrast, the PL excited below the band gap ( $2 p$ excitation) is generated throughout the entire medium because of the very long light penetration depth, and it mainly reflects the bulk properties. For comparison, we conducted PL experiments on a polycrystalline film under the same experimental conditions, and only the peak centered at $540 \mathrm{~nm}$ was recorded. Therefore, it is evident from the optical measurements that the aged surface behaves like a typical polycrystalline film, which is also consistent with the XPS and XRD data presented in Figure 1.

The broadening of the emission spectra of the film compared to those of the aged surface may come from different degrees of hydration and/or different hydrogen bonding interactions between the water molecules and the organic cation $\left(\mathrm{CH}_{3} \mathrm{NH}_{3}^{+}\right)$and halide ions. ${ }^{47}$ We measured radiative carrier recombinations of the aged surface and crystal bulk using the time-correlated single-photon counting (TCSPC) technique (Figure S2) and found that the average carrier lifetimes at the aged surface $(2.5 \mathrm{~ns})$ and in the polycrystalline film $(3 \mathrm{~ns})$ were more than 2 orders of magnitude shorter than the carrier lifetime of the crystal bulk (360 ns). The large difference between the two lifetimes on surfaces and in bulk crystals can be attributed to the change of defect states, in which defect states are predominately present at the surfaces because of the hydration and surface disorder. Additionally, the carrier diffusion on the surface can be also responsible for the shorter lifetime on the surface. As shown in Figure 2b,c, optical band gap experiments surprisingly showed that the absorption features and band edges of the aged surface and the film were 2.27 and $2.28 \mathrm{eV}$, respectively, which we attributed to the compression of the lattice; however, it was $2.22 \mathrm{eV}$ for the pristine surface, again confirming the XRD, steady-state, and time-resolved PL results. The unusual band gap for the pristine surface may arise from the strain-induced symmetry changes and the character of the orbitals. ${ }^{48}$ As evidenced from the STM results (shown later), a tilt in the octahedral by changing the metal-halide-metal angle, clearly suggests strain incorporated into the bulk-crystal lattice. This further fortifies the unusual band edge behavior in the surface and bulk in the present case. Additionally, the unusual band gaps can also arise from the large thickness of the crystal in addition to the ordered and disordered domains. ${ }^{49}$ On the other hand, the transient absorption experiments (Figure 2d) with $1 \mathrm{P}$ excitation showed ground-state bleaching at $520 \mathrm{~nm}$, which is consistent with the absorption spectra of the film and the aged surface, providing 
another strong piece of evidence of the similarity between the aged surface and the polycrystalline film. Similar optical properties, such as emission and lifetimes, were also observed on the aged surface and crystal bulk of $\mathrm{FAPbBr}_{3}$ (FA = formamidinium) single crystal (Figure S3).

To validate our hypothesis, we fabricated a simple visible photodetector with an $\mathrm{Au} / \mathrm{MAPbBr}_{3} / \mathrm{Au}$ configuration. This photodetector distinctly showed a higher photocurrent from the pristine surface and a lower photocurrent from the aged surface under air mass (AM) $1.5 \mathrm{G}$ illumination (Figure 2e). We attributed this result to the interfacial contact resistance resulting from hydrate formation, which lowers the net carrier collection.

We also expected that the performance of a solar cell would depend on the $\mathrm{RH}$ of the ambient air because of the hygroscopic nature of organolead halide perovskites. A typical hole-transporter free solar cell on a $6 \mu \mathrm{m}$ thick $\mathrm{MAPbBr}_{3}$ single crystal demonstrated 4.24\% PCE (Figure 2f) with impressively high $V_{\mathrm{OC}}$ of $1.2 \mathrm{~V}$. This high $V_{\mathrm{OC}}$ can be attributed to the full depletion of electrons, within a depletion width of $7 \mu \mathrm{m}$, implying that the photoexcited carriers easily drift to fluorinedoped tin oxide and Au electrodes. ${ }^{50}$ The complexity of cleaving a $6 \mu \mathrm{m}$ crystal prompted us to try the alternative approach of subjecting the as-fabricated device to high ( $\geq 55 \%)$ $\mathrm{RH}$ conditions and monitoring the degradation over a few days of slower hydrate formation from the top Au electrode. In the initial 2 days, we observed a small drop in PCE of 1\%, whereas prolonged exposure led to a drop in PCE of $\sim 13 \%$ and $\sim 17 \%$ on the sixth and tenth days, respectively, which we attributed to surface hydration (Table S2). Interestingly, recent studies showed that monohydrate formation lowers device performance, ${ }^{30}$ which can be nearly reversible upon dehydration, as the consequential formation of $\mathrm{PbX}_{6}$-based byproducts is partially reversible. $^{51} \mathrm{We}$ observed that the formation of polyhydrate upon exposure of the perovskite surfaces to high levels of moisture led to the formation of hydrate phases that significantly lowered device performance. Moreover, under extreme hydration conditions, weakening of the hydrogen bonding between $\mathrm{PbX}_{6}$ and $\mathrm{MA}$ cations may also lead to an irreversible change and hence to a complete failure of the device. ${ }^{52}$ Similar studies on $\mathrm{CH}_{3} \mathrm{NH}_{3} \mathrm{PbI}_{3-x} \mathrm{Cl}_{x}$ perovskite thinfilm solar cells showed that the initial PCE of less than $4 \%$ dropped to less than $2 \%$ because of moisture that was ascribed to interface degradation. ${ }^{53}$ While films grown under $30 \% \mathrm{RH}$ in ambient air compared with a film grown under dry conditions ${ }^{18}$ showed enhanced optoelectronic properties, we observed that the single crystals grown at $\mathrm{RH} \geq 55 \%$ clearly exhibited detrimental degradation effects on device performance. A more detailed analysis of perovskite surface transformation due to hydration is presented below.

Cross-sectional focused ion beam scanning electron microscope (FIB-SEM) images (Figures 3a, S4, and S5) clearly show the different morphologies of aged and pristine crystal surfaces. Hydration of the perovskite leaves a clear signature of morphological change by forming various types of grains. ${ }^{54}$ Crystal surfaces are prone to degrade under high $\mathrm{RH}$ conditions; other factors such as disordering of MA cation can also contribute to the surface traps and hence the surface transformation. Therefore, it is reasonable to suggest that lattice deformation arises from the strain at the surface, resulting in the observed larger bandgaps at the surface $(2.27 \mathrm{eV})$ compared to in the bulk $(2.22 \mathrm{eV})$ of the single crystal. The FIB-SEM measurements confirm that perovskite hydration results in the clear contrast between the morphologies at the aged and pristine surfaces.

We thus further investigated the surface transformation of aged and pristine surfaces at higher resolution by performing STM under UHV conditions. Special care was taken to account for tip-related artifacts by systematically scanning from large to small areas and from backward to forward on all surfaces (Figures S6-S8). Topographic STM images of the aged surface reveal significant clustering and mounding throughout the surface (Figure $3 b, c$ ). This is not surprising as a perovskite hydrate crystal is substantially swollen by lattice expansion of $6 \%$, indicating that hydration is accompanied by considerable volume expansion of the perovskite unit cell, which results in local stress and the need for relaxation of the surface structure. $^{30}$ This may account for the polycrystalline appearance of the aged surface. On the other hand, a pristine surface cleaved parallel to one facet of the crystal reveals the crystalline nature of the material, with layer-after-layer stacking as expected in a crystalline material, as shown in Figure 3d,e. Nevertheless, there are also substantial defects in the pristine surface, suggestive of the imperfect cleaving of the perovskite crystal.

The small-scale STM topographic image also shows an ordered structure with some regular bright protrusions, as displayed in Figure 3f,h. The density of states (DOS) in all energy levels above the conduction band in halide perovskites involves the $\mathrm{Pb} 6 \mathrm{~s}$ and $6 \mathrm{p}$ orbitals. ${ }^{55}$ The ordered bright protrusions in the STM image (Figure $3 \mathrm{f}$ ) captured at positive voltages $(2.5 \mathrm{~V})$ are thus the manifestation of $\mathrm{Pb}$-rich octahedra with $\mathrm{a} \mathrm{Pb}$ atom at the center.

In a closer view, ordering of the bright protrusions is found to be distorted from an ideal crystal structure, which can be attributed to the lattice strain forcing the octahedra to tilt by changing the metal-halide-metal angle. ${ }^{56}$ It is reasonable to conclude that the distance measured between the two $\mathrm{Pb}$ atoms $(1.48 \mathrm{~nm})$ from the line graph and marked in Figure $3 \mathrm{~g}$ (also see Figure S9) corresponds to the diagonal distance $(1.45 \mathrm{~nm})$ between $\mathrm{Pb}$ atoms in the (110) plane. The apparent step height of each layer was obtained by a line profile, as indicated by the straight line in Figure $3 \mathrm{~h}$. Each layer has an apparent height of $0.52 \pm 0.02 \mathrm{~nm}$ (Figure 3i). Mismatching between the measured height and the interplanar spacing $(\sqrt{ } 2 a=0.84$ $\mathrm{nm}$ ) along the (110) plane may result from lattice-straininduced reconstruction from the tilting octahedra and a subsequent change in the electronic band structure near the band edges, as described elsewhere. ${ }^{56}$ Controlled experiments were carried out by exposing the pristine surface to ambient air overnight for further XPS and STM analyses. The XPS O 1s core level peak clearly exhibits signatures of hydration.

The observed shoulders at 532.5 and $533.3 \mathrm{eV}$ (insets to Figure 1e,g) indicate the respective incorporation of molecular and multilayer water into the perovskite crystal's surface. ${ }^{43}$ Our analysis reveals the formation of clusters on the exposed pristine surface which are similar in appearance to those formed on the aged surface of as-grown crystals (Figure 3j,1). After exposure of the pristine surface to ambient air, the hydration found on the crystalline surfaces corroborates the hygroscopic nature of the surfaces of organolead halide perovskites when exposed to ambient moisture over time. Exposure of the pristine surface to high levels of moisture therefore leads to degradation of the perovskite and to the formation of hydrate phases. $^{30}$ 
The presence of this perovskite hydrate phase is also confirmed by the $\mathrm{O} 1 \mathrm{~s}$ peak in the XPS image, which shows the main component around $532 \mathrm{eV}$ corresponding to the $\mathrm{O}-\mathrm{H}$ species discussed above. Hydroxyl radicals and hydroxide ions formed via water dissociation were found to interact with hydrogens/protons from the top-layer MA cations upon adsorption, thus forming perovskite hydrate on the crystalline perovskite surface, which causes surface strain due to lattice expansion. ${ }^{30,57}$ The perovskite hydrate formed on the pristine surface due to exposure to ambient air can be treated as a model system of epitaxial growth of perovskite hydrate on a crystalline perovskite surface. In this model, the epitaxial film suffers from tensile strain induced by the lattice mismatch with the pristine surface. As the epitaxial film grows, defects and nanostructures formed on the surface become possible pathways to release the strain stored in the epitaxial film, which has also been observed in $\mathrm{Ge} / \mathrm{Si}$ heteroepitaxial systems. ${ }^{58}$ The distending one-dimensional (1D) bright lines marked by rectangles in Figure $3 j$ are evidence of the formation of epitaxially grown perovskite hydrate. The average dimensions of these lines are $12 \mathrm{~nm} \times 5 \mathrm{~nm} \times 1.2 \mathrm{~nm}$ when measured along the marked lines shown in Figure $3 j$ by extracting the line profiles, as indicated by the black and green lines in Figure $3 \mathrm{k}$.

The nanostructures as indicated by a ring of bright spots with a dark spot in the center, as shown in Figure $3 \mathbf{j}$, at various random positions can be attributed to a hopping mechanism. ${ }^{59}$ Our observation of a ring that clusters on the exposed pristine surface of perovskite specimens, which possibly could be perovskite hydrate, is in accordance with previously reported clustering observations on various electrically conductive surfaces. ${ }^{60-63}$ This accumulated evidence suggests that the lattice strain on the top aged surface is the impetus for water incorporation into the perovskite, leading to the formation of these nanostructures. We therefore attribute these polycrystalline features and grain boundaries (Figure S10) to surface disorder on the aged surface modulated by exposure to moisture and conceivably oxygen. The morphological variations of the pristine and aged crystal surface could possibly originate from the growth process and crystal collection process; however, we have carried out control experiments and observed the emergence of STM and XPS features on the pristine surface that are similar to those of the aged surface during its exposure in the air. More specifically, the control experiments confirmed the gradual convergence of the features of the pristine surface during exposure in the air with those of the aged crystal surface by showing similar $O$ 1s peaks in the XPS spectra and protrusion formation in STM images. Therefore, we believe these results are suggestive of hydration-induced morphological changes.

Understanding the surface properties of perovskite single crystals, including their surface qualities, stability, and carrier recombination, is essential to improving the ambient operation and optimization of devices based on these crystals. Here, we directly visualized the surface transformation of perovskite single crystals using state-of-the-art spectroscopy and microscopy techniques at atomic spatial resolution. Our results clearly indicate the formation of a hydrated layer (polycrystalline-like structure) on the surfaces of single crystals and hence the formation of grain boundaries. Several pieces of evidence from our array of experiments show that the incipient surface of a single crystal has a different optical band gap, carrier recombination, excitation spectra, PL spectral position, and lifetime characteristics compared with a pristinely cleaved crystal surface. This observation clearly demonstrates why the aged surfaces of these crystals behave uniquely in contrast with the behaviors of bulk single crystals. These findings provide new insights into the surface transformation of a single crystal that are consequential to optimizing the surface quality and stability of perovskite single crystals for the further amelioration of photovoltaic and optoelectronic devices.

\section{ASSOCIATED CONTENT}

\section{S Supporting Information}

The Supporting Information is available free of charge on the ACS Publications website at DOI: 10.1021/acsenergylett.6b00517.

Figures showing the experimental setup of cleaving single crystal, optical measurements of $\mathrm{FAPbBr}_{3}$ single crystal, and SEM investigations and STM topographies with the chronological history of the scans; tables showing the chemical compositions and device statistics (All data needed to evaluate the conclusions in the Letter are present in the text and/or the Supporting Information. Additional data related to this Letter may be requested from the authors.) (PDF)

\section{AUTHOR INFORMATION}

\section{Corresponding Authors}

*E-mail: omar.abdelsaboor@kaust.edu.sa.

*E-mail: osman.bakr@kaust.edu.sa.

*E-mail: aram.amassian@kaust.edu.sa.

ORCID

Emre Yengel: 0000-0001-7208-4803

Omar F. Mohammed: 0000-0001-8500-1130

Present Address

†A.L.A.: Nanochemistry Department, Istituto Italiano di Tecnologia, Via Morego 30, 16163 Genova, Italy.

\section{Notes}

The authors declare no competing financial interest.

\section{ACKNOWLEDGMENTS}

King Abdullah University of Science and Technology (KAUST) supported the work reported here. The authors gratefully acknowledge funding support from KAUST and from King Abdul-Aziz City for Science and Technology, Technology Innovation Center for Solid-State Lighting at KAUST. We also acknowledge Ahmed M. El-Zohry and Chen Yang for their help in the discussions related to reabsorption and crystal synthesis.

\section{REFERENCES}

(1) Manser, J. S.; Saidaminov, M. I.; Christians, J. A.; Bakr, O. M.; Kamat, P. V. Making and Breaking of Lead Halide Perovskites. Acc. Chem. Res. 2016, 49, 330-338.

(2) Tsai, H.; Nie, W.; Blancon, J.-C.; Stoumpos, C. C.; Asadpour, R.; Harutyunyan, B.; Neukirch, A. J.; Verduzco, R.; Crochet, J. J.; Tretiak, S.; et al. High-Efficiency Two-Dimensional Ruddlesden-Popper Perovskite Solar Cells. Nature 2016, 536, 312-316.

(3) Neukirch, A. J.; Nie, W.; Blancon, J.-C.; Appavoo, K.; Tsai, H.; Sfeir, M. Y.; Katan, C.; Pedesseau, L.; Even, J.; Crochet, J. J.; et al. Polaron Stabilization by Cooperative Lattice Distortion and Cation Rotations in Hybrid Perovskite Materials. Nano Lett. 2016, 16, 38093816.

(4) Mao, L.; Tsai, H.; Nie, W.; Ma, L.; Im, J.; Stoumpos, C. C.; Malliakas, C. D.; Hao, F.; Wasielewski, M. R.; Mohite, A. D.; et al. Role 
of Organic Counterion in Lead- and Tin-based Two-dimensional Semiconducting Iodide Perovskites and Application in Planar Solar Cells. Chem. Mater. 2016. DOI: 10.1021/acs.chemmater.6b03054.

(5) Green, M. A.; Emery, K.; Hishikawa, Y.; Warta, W.; Dunlop, E. D. Solar Cell Efficiency Tables (version 47). Prog. Photovoltaics 2016, 24, $3-11$.

(6) Zhumekenov, A. A.; Saidaminov, M. I.; Haque, M. A.; Alarousu, E.; Sarmah, S. P.; Murali, B.; Dursun, I.; Miao, X.-H.; Abdelhady, A. L.; $\mathrm{Wu}, \mathrm{T}$.; et al. Formamidinium Lead Halide Perovskite Crystals with Unprecedented Long Carrier Dynamics and Diffusion Length. ACS Energy Lett. 2016, 1, 32-37.

(7) Lin, Q.; Armin, A.; Burn, P. L.; Meredith, P. Filterless Narrowband Visible Photodetectors. Nat. Photonics 2015, 9, 687-694.

(8) Yang, Y.; Yang, M.; Zhu, K.; Johnson, J. C.; Berry, J. J.; van de Lagemaat, J.; Beard, M. C. Large Polarization-Dependent Exciton Optical Stark Effect in Lead Iodide Perovskites. Nat. Commun. 2016, 7, 12613.

(9) Yang, Y.; Ostrowski, D. P.; France, R. M.; Zhu, K.; van de Lagemaat, J.; Luther, J. M.; Beard, M. C. Observation of a Hot-Phonon Bottleneck in Lead-Iodide Perovskites. Nat. Photonics 2016, 10, 5359.

(10) Fang, H.-H.; Raissa, R.; Abdu-Aguye, M.; Adjokatse, S.; Blake, G. R.; Even, J.; Loi, M. A. Hybrid Perovskites: Photophysics of Organic-Inorganic Hybrid Lead Iodide Perovskite Single Crystals. Adv. Funct. Mater. 2015, 25, 2346-2346.

(11) Dong, Q.; Fang, Y.; Shao, Y.; Mulligan, P.; Qiu, J.; Cao, L.; Huang, J. Electron-Hole Diffusion Lengths $>175 \mu \mathrm{m}$ in SolutionGrown $\mathrm{CH}_{3} \mathrm{NH}_{3} \mathrm{PbI}_{3}$ Single Crystals. Science 2015, 347, 967-970.

(12) Pan, J.; Sarmah, S. P.; Murali, B.; Dursun, I.; Peng, W.; Parida, M. R.; Liu, J.; Sinatra, L.; Alyami, N.; Zhao, C.; et al. Air-Stable Surface-Passivated Perovskite Quantum Dots for Ultra-Robust, Singleand Two-Photon-Induced Amplified Spontaneous Emission. J. Phys. Chem. Lett. 2015, 6, 5027-5033.

(13) Yang, Y.; Yan, Y.; Yang, M.; Choi, S.; Zhu, K.; Luther, J. M.; Beard, M. C. Low Surface Recombination Velocity in Solution-Grown $\mathrm{CH}_{3} \mathrm{NH}_{3} \mathrm{PbBr}_{3}$ Perovskite Single Crystal. Nat. Commun. 2015, 6, 7961.

(14) McMeekin, D. P.; Sadoughi, G.; Rehman, W.; Eperon, G. E.; Saliba, M.; Hörantner, M. T.; Haghighirad, A.; Sakai, N.; Korte, L.; Rech, B.; et al. A Mixed-Cation Lead Mixed-Halide Perovskite Absorber for Tandem Solar Cells. Science 2016, 351, 151-155.

(15) Saidaminov, M. I.; Almutlaq, J.; Sarmah, S.; Dursun, I.; Zhumekenov, A. A.; Begum, R.; Pan, J.; Cho, N.; Mohammed, O. F.; Bakr, O. M. Pure $\mathrm{Cs}_{4} \mathrm{PbBr}_{6}$ : Highly Luminescent Zero-Dimensional Perovskite Solids. ACS Energy Lett. 2016, 1, 840-845.

(16) Ahmed, G. H.; Liu, J.; Parida, M. R.; Murali, B.; Bose, R.; AlYami, N. M.; Hedhili, M. N.; Peng, W.; Pan, J.; Besong, T. M. D.; et al. Shape-Tunable Charge Carrier Dynamics at the Interfaces between Perovskite Nanocrystals and Molecular Acceptors. J. Phys. Chem. Lett. 2016, 7, 3913-3919.

(17) Beard, M. C.; Blackburn, J. L.; Johnson, J. C.; Rumbles, G. Status and Prognosis of Future-Generation Photoconversion to Photovoltaics and Solar Fuels. ACS Energy Lett. 2016, 1, 344-347.

(18) Zhou, H.; Chen, Q.; Li, G.; Luo, S.; Song, T.-b.; Duan, H.-S.; Hong, Z.; You, J.; Liu, Y.; Yang, Y. Interface Engineering of Highly Efficient Perovskite Solar Cells. Science 2014, 345, 542-546.

(19) Cho, H.; Jeong, S.-H.; Park, M.-H.; Kim, Y.-H.; Wolf, C.; Lee, C.-L.; Heo, J. H.; Sadhanala, A.; Myoung, N.; Yoo, S.; et al. Overcoming the Electroluminescence Efficiency Limitations of Perovskite Light-Emitting Diodes. Science 2015, 350, 1222-1225.

(20) Zhu, H.; Fu, Y.; Meng, F.; Wu, X.; Gong, Z.; Ding, Q.; Gustafsson, M. V.; Trinh, M. T.; Jin, S.; Zhu, X. Y. Lead Halide Perovskite Nanowire Lasers with Low Lasing Thresholds and High Quality Factors. Nat. Mater. 2015, 14, 636-642.

(21) Bi, D.; Tress, W.; Dar, M. I.; Gao, P.; Luo, J.; Renevier, C.; Schenk, K.; Abate, A.; Giordano, F.; Correa Baena, J.-P.; et al. Efficient Luminescent Solar Cells Based on Tailored Mixed-Cation Perovskites. Sci. Adv. 2016, 2, e1501170.
(22) Murali, B.; Saidaminov, M. I.; Abdelhady, A. L.; Peng, W.; Liu, J.; Pan, J.; Bakr, O. M.; Mohammed, O. F. Robust and Air-Stable Sandwiched Organo-Lead Halide Perovskites for Photodetector Applications. J. Mater. Chem. C 2016, 4, 2545-2552.

(23) Ling, Y.; Yuan, Z.; Tian, Y.; Wang, X.; Wang, J. C.; Xin, Y.; Hanson, K.; Ma, B.; Gao, H. Bright Light-Emitting Diodes Based on Organometal Halide Perovskite Nanoplatelets. Adv. Mater. 2016, 28, 305-311.

(24) Gong, J.; Yang, M.; Ma, X.; Schaller, R. D.; Liu, G.; Kong, L.; Yang, Y.; Beard, M. C.; Lesslie, M.; Dai, Y.; et al. Electron-Rotor Interaction in Organic-Inorganic Lead Iodide Perovskites Discovered by Isotope Effects. J. Phys. Chem. Lett. 2016, 7, 2879-2887.

(25) Yoon, S. J.; Draguta, S.; Manser, J. S.; Sharia, O.; Schneider, W. F.; Kuno, M.; Kamat, P. V. Tracking Iodide and Bromide Ion Segregation in Mixed Halide Lead Perovskites during Photoirradiation. ACS Energy Lett. 2016, 1, 290-296.

(26) Nie, W.; Tsai, H.; Asadpour, R.; Blancon, J.-C.; Neukirch, A. J.; Gupta, G.; Crochet, J. J.; Chhowalla, M.; Tretiak, S.; Alam, M. A.; et al. High-Efficiency Solution-Processed Perovskite Solar Cells with Millimeter-Scale Grains. Science 2015, 347, 522-525.

(27) Manser, J. S.; Christians, J. A.; Kamat, P. V. Intriguing Optoelectronic Properties of Metal Halide Perovskites. Chem. Rev. 2016. DOI:10.1021/acs.chemrev.6b00136.

(28) Shi, D.; Adinolfi, V.; Comin, R.; Yuan, M.; Alarousu, E.; Buin, A.; Chen, Y.; Hoogland, S.; Rothenberger, A.; Katsiev, K.; et al. Low Trap-State Density and Long Carrier Diffusion in Organolead Trihalide Perovskite Single Crystals. Science 2015, 347, 519-522.

(29) Abdelhady, A. L.; Saidaminov, M. I.; Murali, B.; Adinolfi, V.; Voznyy, O.; Katsiev, K.; Alarousu, E.; Comin, R.; Dursun, I.; Sinatra, L.; et al. Heterovalent Dopant Incorporation for Bandgap and Type Engineering of Perovskite Crystals. J. Phys. Chem. Lett. 2016, 7, 295301.

(30) Leguy, A. M. A.; Hu, Y.; Campoy-Quiles, M.; Alonso, M. I.; Weber, O. J.; Azarhoosh, P.; van Schilfgaarde, M.; Weller, M. T.; Bein, T.; Nelson, J.; et al. Reversible Hydration of $\mathrm{CH}_{3} \mathrm{NH}_{3} \mathrm{PbI}_{3}$ in Films, Single Crystals, and Solar Cells. Chem. Mater. 2015, 27, 3397-3407.

(31) Gangishetty, M. K.; Scott, R. W. J.; Kelly, T. L. Effect of Relative Humidity on Crystal Growth, Device Performance and Hysteresis in Planar Heterojunction Perovskite Solar Cells. Nanoscale 2016, 8, 6300-6307.

(32) Yang, J.; Siempelkamp, B. D.; Liu, D.; Kelly, T. L. Investigation of $\mathrm{CH}_{3} \mathrm{NH}_{3} \mathrm{PbI}_{3}$ Degradation Rates and Mechanisms in Controlled Humidity Environments Using in Situ Techniques. ACS Nano 2015, 9, 1955-1963.

(33) Wu, B.; Nguyen, H. T.; Ku, Z.; Han, G.; Giovanni, D.; Mathews, N.; Fan, H. J.; Sum, T. C. Discerning the Surface and Bulk Recombination Kinetics of Organic-Inorganic Halide Perovskite Single Crystals. Adv. Energy Mater. 2016, 6, 1600551.

(34) Eperon, G. E.; Habisreutinger, S. N.; Leijtens, T.; Bruijnaers, B. J.; van Franeker, J. J.; deQuilettes, D. W.; Pathak, S.; Sutton, R. J.; Grancini, G.; Ginger, D. S.; et al. The Importance of Moisture in Hybrid Lead Halide Perovskite Thin Film Fabrication. ACS Nano 2015, 9, 9380-9393.

(35) Fang, H.-H.; Adjokatse, S.; Wei, H.; Yang, J.; Blake, G. R.; Huang, J.; Even, J.; Loi, M. A. Ultrahigh Sensitivity of Methylammonium Lead Tribromide Perovskite Single Crystals to Environmental Gases. Sci. Adv. 2016, 2, e1600534.

(36) Zhao, L.; Kerner, R. A.; Xiao, Z.; Lin, Y. L.; Lee, K. M.; Schwartz, J.; Rand, B. P. Redox Chemistry Dominates the Degradation and Decomposition of Metal Halide Perovskite Optoelectronic Devices. ACS Energy Lett. 2016, 1, 595-602.

(37) Petrus, M. L.; Hu, Y.; Moia, D.; Calado, P.; Leguy, A. M. A.; Barnes, P. R. F.; Docampo, P. The Influence of Water Vapor on the Stability and Processing of Hybrid Perovskite Solar Cells Made from Non-Stoichiometric Precursor Mixtures. ChemSusChem 2016, 9, 2699-2707.

(38) Saidaminov, M. I.; Abdelhady, A. L.; Murali, B.; Alarousu, E.; Burlakov, V. M.; Peng, W.; Dursun, I.; Wang, L.; He, Y.; Maculan, G.; et al. High-Quality Bulk Hybrid Perovskite Single Crystals within 
Minutes by Inverse Temperature Crystallization. Nat. Commun. 2015, 6, 7586 .

(39) Oesinghaus, L.; Schlipf, J.; Giesbrecht, N.; Song, L.; Hu, Y.; Bein, T.; Docampo, P.; Müller-Buschbaum, P. Toward Tailored Film Morphologies: The Origin of Crystal Orientation in Hybrid Perovskite Thin Films. Adv. Mater. Interfaces 2016, 3, 1600403.

(40) Giesbrecht, N.; Schlipf, J.; Oesinghaus, L.; Binek, A.; Bein, T.; Müller-Buschbaum, P.; Docampo, P. Synthesis of Perfectly Oriented and Micrometer-Sized $\mathrm{MAPbBr}_{3}$ Perovskite Crystals for Thin-Film Photovoltaic Applications. ACS Energy Lett. 2016, 1, 150-154.

(41) Bagri, A.; Mattevi, C.; Acik, M.; Chabal, Y. J.; Chhowalla, M.; Shenoy, V. B. Structural Evolution During the Reduction of Chemically Derived Graphene Oxide. Nat. Chem. 2010, 2, 581-587.

(42) Lu, J.; Lei, Y.; Lau, K. C.; Luo, X.; Du, P.; Wen, J.; Assary, R. S.; Das, U.; Miller, D. J.; Elam, J. W.; et al. A Nanostructured Cathode Architecture for Low Charge Overpotential in Lithium-Oxygen Batteries. Nat. Commun. 2013, 4, 2383.

(43) Halwidl, D.; Stoger, B.; Mayr-Schmolzer, W.; Pavelec, J.; Fobes, D.; Peng, J.; Mao, Z.; Parkinson, G. S.; Schmid, M.; Mittendorfer, F.; et al. Adsorption of Water at the SrO Surface of Ruthenates. Nat. Mater. 2015, 15, 450-455.

(44) Yakunin, S.; Protesescu, L.; Krieg, F.; Bodnarchuk, M. I.; Nedelcu, G.; Humer, M.; De Luca, G.; Fiebig, M.; Heiss, W.; Kovalenko, M. V. Low-Threshold Amplified Spontaneous Emission and Lasing from Colloidal Nanocrystals of Caesium Lead Halide Perovskites. Nat. Commun. 2015, 6, 8056.

(45) Walters, G.; Sutherland, B. R.; Hoogland, S.; Shi, D.; Comin, R.; Sellan, D. P.; Bakr, O. M.; Sargent, E. H. Two-Photon Absorption in Organometallic Bromide Perovskites. ACS Nano 2015, 9, 9340-9346.

(46) Yang, Y.; Yang, M.; Li, Z.; Crisp, R.; Zhu, K.; Beard, M. C. Comparison of Recombination Dynamics in $\mathrm{CH}_{3} \mathrm{NH}_{3} \mathrm{PbBr}_{3}$ and $\mathrm{CH}_{3} \mathrm{NH}_{3} \mathrm{PbI}_{3}$ Perovskite Films: Influence of Exciton Binding Energy. J. Phys. Chem. Lett. 2015, 6, 4688-4692.

(47) Grancini, G.; D’Innocenzo, V.; Dohner, E. R.; Martino, N.; Srimath Kandada, A. R.; Mosconi, E.; De Angelis, F.; Karunadasa, H. I.; Hoke, E. T.; Petrozza, A. $\mathrm{CH}_{3} \mathrm{NH}_{3} \mathrm{PbI}_{3}$ Perovskite Single Crystals: Surface Photophysics and their Interaction with the Environment. Chem. Sci. 2015, 6 (12), 7305-7310.

(48) Grote, C.; Berger, R. F. Strain Tuning of Tin-Halide and Lead-Halide Perovskites: A First-Principles Atomic and Electronic Structure Study. J. Phys. Chem. C 2015, 119, 22832-22837.

(49) Dar, M. I.; Jacopin, G.; Meloni, S.; Mattoni, A.; Arora, N.; Boziki, A.; Zakeeruddin, S. M.; Rothlisberger, U.; Grätzel, M. Origin of Unusual Bandgap Shift and Dual Emission in Organic-Inorganic Lead Halide Perovskites. Science Adv. 2016, 2, e1601156.

(50) Peng, W.; Wang, L.; Murali, B.; Ho, K.-T.; Bera, A.; Cho, N.; Kang, C.-F.; Burlakov, V. M.; Pan, J.; Sinatra, L.; et al. Solution-Grown Monocrystalline Hybrid Perovskite Films for Hole-Transporter-Free Solar Cells. Adv. Mater. 2016, 28, 3383-3390.

(51) Dong, X.; Fang, X.; Lv, M.; Lin, B.; Zhang, S.; Ding, J.; Yuan, N. Improvement of the Humidity Stability of Organic-Inorganic Perovskite Solar Cells using Ultrathin $\mathrm{Al}_{2} \mathrm{O}_{3}$ Layers Prepared by Atomic Layer Deposition. J. Mater. Chem. A 2015, 3, 5360-5367.

(52) Berhe, T. A.; Su, W.-N.; Chen, C.-H.; Pan, C.-J.; Cheng, J.-H.; Chen, H.-M.; Tsai, M.-C.; Chen, L.-Y.; Dubale, A. A.; Hwang, B.-J. Organometal Halide Perovskite Solar Cells: Degradation and Stability. Energy Environ. Sci. 2016, 9, 323-356.

(53) Kaltenbrunner, M.; Adam, G.; Glowacki, E. D.; Drack, M.; Schwodiauer, R.; Leonat, L.; Apaydin, D. H.; Groiss, H.; Scharber, M. C.; White, M. S.; et al. Flexible High Power-Per-Weight Perovskite Solar Cells with Chromium Oxide-Metal Contacts for Improved Stability in Air. Nat. Mater. 2015, 14, 1032-1039.

(54) Zhao, J.; Cai, B.; Luo, Z.; Dong, Y.; Zhang, Y.; Xu, H.; Hong, B.; Yang, Y.; Li, L.; Zhang, W.; et al. Investigation of the Hydrolysis of Perovskite Organometallic Halide $\mathrm{CH}_{3} \mathrm{NH}_{3} \mathrm{PbI}_{3}$ in Humidity Environment. Sci. Rep. 2016, 6, 21976.

(55) Guerrero, A.; You, J.; Aranda, C.; Kang, Y. S.; Garcia-Belmonte, G.; Zhou, H.; Bisquert, J.; Yang, Y. Interfacial Degradation of Planar Lead Halide Perovskite Solar Cells. ACS Nano 2016, 10, 218-224.
(56) Azpiroz, J. M.; Mosconi, E.; Bisquert, J.; De Angelis, F. Defect Migration in Methylammonium Lead Iodide and its Role in Perovskite Solar Cell Operation. Energy Environ. Sci. 2015, 8, 2118-2127.

(57) Grancini, G.; Srimath Kandada, A. R.; Frost, J. M.; Barker, A. J.; De Bastiani, M.; Gandini, M.; Marras, S.; Lanzani, G.; Walsh, A.; Petrozza, A. Role of Microstructure in the Electron-Hole Interaction of Hybrid Lead Halide Perovskites. Nat. Photonics 2015, 9, 695-701.

(58) Jishi, R. A.; Ta, O. B.; Sharif, A. A. Modeling of Lead Halide Perovskites for Photovoltaic Applications. J. Phys. Chem. C 2014, 118, 28344-28349.

(59) Amat, A.; Mosconi, E.; Ronca, E.; Quarti, C.; Umari, P.; Nazeeruddin, M. K.; Grätzel, M.; De Angelis, F. Cation-Induced BandGap Tuning in Organohalide Perovskites: Interplay of Spin-Orbit Coupling and Octahedra Tilting. Nano Lett. 2014, 14, 3608-3616.

(60) Zhang, L.; Sit, P. H. L. Ab Initio Study of Interaction of Water, Hydroxyl Radicals, and Hydroxide Ions with $\mathrm{CH}_{3} \mathrm{NH}_{3} \mathrm{PbI}_{3}$ and $\mathrm{CH}_{3} \mathrm{NH}_{3} \mathrm{PbBr}_{3}$ Surfaces. J. Phys. Chem. C 2015, 119, 22370-22378.

(61) Persichetti, L.; Sgarlata, A.; Fanfoni, M.; Balzarotti, A. Heteroepitaxy of Ge on Singular and Vicinal Si Surfaces: Elastic Field Symmetry and Nanostructure Growth. J. Phys.: Condens. Matter 2015, 27, 253001.

(62) Merte, L. R.; Peng, G.; Bechstein, R.; Rieboldt, F.; Farberow, C. A.; Grabow, L. C.; Kudernatsch, W.; Wendt, S.; Lægsgaard, E.; Mavrikakis, M.; et al. Water-Mediated Proton Hopping on an Iron Oxide Surface. Science 2012, 336, 889-893.

(63) Michaelides, A.; Morgenstern, K. Ice Nanoclusters at Hydrophobic Metal Surfaces. Nat. Mater. 2007, 6, 597-601. 